\title{
Erratum
}

Z. Phys. D-Atoms, Molecules and Clusters 12, 353-356(1991)

\section{Static electric dipole polarizability of small sodium aggregates}

\author{
I. Moullet ${ }^{1}$, José Luís Martins ${ }^{2}$, F. Reuse ${ }^{1}$, and J. Buttet ${ }^{1}$ \\ ${ }^{1}$ Institut de Physique Expérimentale, École Polytechnique Fédérale de Lausanne, CH-1015 Lausanne, \\ Switzerland \\ ${ }^{2}$ Department of Chemical Engineering and Materials Science, University of Minnesota, Minneapolis, \\ MN 55455, USA
}

Received 6 February 1991

The values for the averaged molecular polarizabilities of $\mathrm{Na}_{2}$ and $\mathrm{Na}_{3}$ reported in Table 3 are incorrect due to a missing conversion factor in the evaluation of the vibrational contributions. The correct values evaluated with better accuracy are reported in the last Table of [1]. We had concluded that the vibrational were small. The new values show that they are actually negligible.

\section{References}

1. Moullet, I., et al.: Phys. Rev. B42, 11598 (1990) 